\title{
EXISTENCIA Y UNICIDAD DE SOLUCIONES REGULARES DE LA ECUACIÓN DE ONDA SEMILINEAL CON DISIPACIÓN LOCALIZADA EN $\mathbb{R}^{n}$
}

\author{
Pérez Salvatierra Alfonso ${ }^{1}$, Peña Miranda Carlos Alberto ${ }^{2}$
}

Resumen: El presente artículo es parte de los resultados de la tesis titulada: Comportamiento asintótico para la ecuación de onda semilineal con amortiguamiento local en dominios no acotado, en este artículo estudiamos la existencia y unicidad de solución regular para la ecuación de onda semilineal con disipación localizada sobre $\mathbb{R}^{n}$ dado por,

$$
\left\{\begin{array}{l}
u_{t t}-\Delta u+\alpha(x) u+f(u)+a(x) u_{t}=0 \text { en } \mathbb{R}^{n} \times(0, \infty), \\
u(0)=u_{0} \in H^{2}\left(\mathbb{R}^{n}\right), u_{t}(0)=u_{1} \in H^{1}\left(\mathbb{R}^{n}\right) .
\end{array}\right.
$$

Palabras Claves: Ecuación de onda semilineal, solución regular, semigrupos de operadores lineales

Abstract: This article is part of the results of the thesis: Asymptotic behavior for semilinear wave equation with local damping in unbounded domains, in this paper we study the existence and uniqueness of smooth solution for the semilinear wave equation with localized dissipation on $\mathbb{R}^{n}$ given by,

$$
\left\{\begin{array}{l}
u_{t t}-\Delta u+\alpha(x) u+f(u)+a(x) u_{t}=0 \text { in } \mathbb{R}^{n} \times(0, \infty), \\
u(0)=u_{0} \in H^{2}\left(\mathbb{R}^{n}\right), u_{t}(0)=u_{1} \in H^{1}\left(\mathbb{R}^{n}\right) .
\end{array}\right.
$$

Key Words: Semilinear wave equation, smooth solution, semigroups of linear operators.

\section{Introducción}

Los trabajos de sistemas con disipación localmente distribuidos son estudiados con bastante interés desde la década de los 90 hasta la fecha, estos sobre dominios $\Omega$ acotados de $\mathbb{R}^{n}$, aplicados a diferentes sistemas como podemos citar: visco elástico, termoelásticos, problemas de contacto, ecuación de Kirchoff, entre otros. Ver [3], [6], [9], [10], [11],[12] y [13].

Los términos disipativos en una ecuación, sirven para garantizar un comportamiento asintótico o polinomial de la energía asociada al sistema planteado.

Este artículo tiene por objetivo estudiar la existencia y unicidad de solución regular para la ecuación semilinear de onda con disipación localizada sobre $\mathbb{R}^{n}$ dado por,

$$
\left\{\begin{array}{l}
u_{t t}-\Delta u+\alpha(x) u+f(u)+a(x) u_{t}=0 \text { en } \mathbb{R}^{n} \times(0,+\infty), \\
u(0)=u_{0} \in H^{2}\left(\mathbb{R}^{n}\right), u_{t}(0)=u_{1} \in H^{1}\left(\mathbb{R}^{n}\right),
\end{array}\right.
$$

con las hipótesis

$$
\text { (H1) } a \in L_{+}^{\infty}\left(\mathbb{R}^{n}\right) ; a(x) \geq a_{0} \text { c.s. en } \Omega_{R}=\mathbb{R}^{n} \backslash B_{R}=\left\{x \in \mathbb{R}^{n} /|x| \geq R\right\}
$$

para $R>0$, con $B_{R}=\left\{x \in \mathbb{R}^{n} /|x|<R\right\}$.

(H2) $f(s) s \geq 0$, para todo $s \in \mathbb{R}$.

\footnotetext{
${ }^{1}$ UNMSM, Lima, Perú, e-mail: aperezs@unmsm.edu.pe

${ }^{2}$ UNMSM, Lima, Perú, e-mail: cpenam@unmsm.edu.pe
} 
(H3) $f \in C^{1}(\mathbb{R})$, satisface la siguiente condición de crecimiento: existe $C>0$, $p>1$, con $(n-2) p \leq n$ tal que

$$
|f(x)-f(y)| \leq C\left(1+|x|^{p-1}+|y|^{p-1}\right)|x-y| \text {, para todo } x, y \in \mathbb{R} \text {. }
$$

(H4) $\alpha \in W^{1 . \infty}\left(\mathbb{R}^{n}\right) ; \alpha(x) \geq \alpha_{0}$ c.s. en $\mathbb{R}^{n}$.

El modelo matemático descrito es estudiado entre otros por E. Zuazua [14] quien prueba con las hipótesis $(\mathrm{H} 1)$ - (H3), la existencia y unicidad de solución débil para

$\alpha(x)=\alpha \in \mathbb{R}$ constante y condiciones iniciales $\left(u_{0}, u_{1}\right) \in H^{1}\left(\mathbb{R}^{n}\right) \times L^{2}\left(\mathbb{R}^{n}\right)$.

\section{Preliminares}

En esta sección presentamos algunos resultados y definiciones necesarias para el estudio de la existencia y unicidad de soluciones regulares de la ecuación (1).

Sea $H$ un espacio de Hilbert sobre $\mathbb{R}$. Denotaremos $(\cdot, \cdot)$ y $\|\cdot\|$ el producto interno y norma respectivamente en $\mathrm{H}$, entonces se tiene las siguientes definiciones:

Definición 2.1. Una familia $\{S(t)\}_{t \geq 0}$ de operadores lineales y continuos $S(t): H \rightarrow H$ se denomina semigrupo de operadores lineales acotado de $X$ si:

i) $S(0)=I$, donde $I$ es el operador identidad.

ii) $S(t+s)=S(t) S(s), \forall t, s \in \mathbb{R}^{+}$.

Diremos que el semigrupo $S$ es de clase $C_{0}$ si

iii) $\lim _{t \rightarrow 0^{+}}\|(S(t)-I) x\|=0, \forall x \in H$.

Teorema 2.2. Sea $\{S(t)\}_{t \geq 0}$ un semigrupo de clase $C_{0}$, entonces existen $w \geq 0$ y $M \geq 1$ tal que

$$
\|S(t)\| \leq M e^{w t}, \forall t \geq 0
$$

Demostración: Ver Pazy A. [7].

Observación 2.3. Tomando $w=0$, el teorema 2.2 afirma que existe una constante $M \geq 1$ tal que $\|S(t)\| \leq M$, para todo $t \geq 0$. En este caso diremos que $\{S(t)\}_{t \geq 0}$ es un semigrupo uniformente acotado de clase $C_{0}$. Además si $w=0$ y $M=1$ diremos que $\{S(t)\}_{t \geq 0}$ es un semigrupo de contracciones de clase $C_{0}$, es decir, $\|S(t)\| \leq 1, \forall t \geq 0$.

Definición 2.4. Sea $\{S(t)\}_{t \geq 0}$ un semigrupo de clase $C_{0}$. El operador lineal $A: D(A) \subset H \rightarrow H$, definido por

$$
D(A)=\left\{x \in H: \lim _{h \rightarrow 0^{+}} \frac{S(h)-I}{h} x \text { existe }\right\} \text { y } A x=\lim _{h \rightarrow 0^{+}} \frac{S(h)-I}{h} x, \forall x \in D(A)
$$

es el generador infinitesimal del semigrupo $\{S(t)\}_{t \geq 0}$ donde $D(A)$ es el dominio de $A$.

Definición 2.5. Sea $A: D(A) \subseteq H \rightarrow H$ un operador lineal no acotado. Diremos que $A$ es monótono si

$$
(A v, v) \geq 0, \forall v \in D(A)
$$

Definición 2.6. Diremos que el operador $A$ es maximal mónotono si $A$ es monótono y también $R(I+A)=H$, es decir, para todo $f \in H$ existe $u \in D(A)$ tal que $u+A u=f$. 
Observación 2.7. Debido al teorema Lumer - Phillips (Pazy [7]) si $-A$ es un operador maximal monótono entonces $-A$ es el generador de un semigrupo de contracciones de clase $C_{0}$.

Entonces el problema de Cauchy abstracto dado por,

$$
\left\{\begin{array}{l}
\frac{d u}{d t}=A u+F(u), \\
u(0)=u_{0},
\end{array}\right.
$$

puede ser estudiado a partir de ahora, sustituyendo $A$ por $-A$.

Es decir, se va a considera el siguiente problema de valor inicial

$$
\left\{\begin{array}{l}
\frac{d u}{d t}+A u=F(u), \\
u(0)=u_{0},
\end{array}\right.
$$

donde $A$ es el generador de un semigrupo de contracciones de clase $C_{0}$ sobre el espacio de Hilbert $H$ y $F: H \rightarrow H$ una función continua.

Definición 2.8. Si $u \in C^{1}([0, T] ; H) \cap C([0, T] ; D(A))$, diremos que u es una solución clásica o fuerte del problema de valor inicial (6).

Observación 2.9. Si $u$ es una solución fuerte de (6), entonces $u$ verifica la ecuación integral dada por

$$
u(t)=S(t) u_{0}+\int_{0}^{t} S(t-s) F(u(s)) d s .
$$

Definición 2.10. Si $u \in C([0, T] ; H)$ verifica el problema de valor inicial (7), diremos que u es una solución débil o generalizada de (6).

Definición 2.11. Diremos que una aplicación $F: H \rightarrow H$ es localmente Lipschitziana, si para cada constante positiva $M$ existe una constante $L_{M}$ tal que

$$
|F(u)-F(v)| \leq L_{M}|u-v|
$$

para todo $u, v \in H$ tal que $|u| \leq M$ y $|v| \leq M$.

Ahora enunciamos el resultado principal de este trabajo

\section{Teorema Central}

Teorema 3.1. Sean $\left(u_{0}, u_{1}\right) \in H^{2}\left(\mathbb{R}^{n}\right) \times H^{1}\left(\mathbb{R}^{n}\right)$ y verificando las hipótesis $(\mathrm{H} 1)-(\mathrm{H} 4)$. Entonces existe una única solución $u:[0,+\infty) \rightarrow \mathbb{R}$ tal que

$$
u \in C\left([0,+\infty) ; H^{2}\left(\mathbb{R}^{n}\right)\right) \cap C^{1}\left([0,+\infty) ; H^{1}\left(\mathbb{R}^{n}\right)\right) \cap C^{2}\left([0,+\infty) ; L^{2}\left(\mathbb{R}^{n}\right)\right) .
$$

\section{Demostración.}

\section{A) Existencia de solución regular}

Consideremos el espacio $H=H^{1}\left(\mathbb{R}^{n}\right) \times L^{2}\left(\mathbb{R}^{n}\right)$ con el producto interno

$$
\left(U_{1}, U_{2}\right)_{H}=\int_{\mathbb{R}^{n}}\left(\nabla u_{1} \cdot \nabla u_{2}+\alpha(x) u_{1} u_{2}+v_{1} v_{2}\right) d x
$$


donde $U_{i}=\left(\begin{array}{c}u_{i} \\ v_{i}\end{array}\right) \in H, i=1,2$

y la norma inducida

$$
\|U\|_{H}=\int_{\mathbb{R}^{n}}\left(|\nabla u|^{2}+\alpha(x)|u|^{2}+|v|^{2}\right) d x
$$

donde $U=\left(\begin{array}{l}u \\ v\end{array}\right)$.

La ecuación (1) puede ser reescrita en la forma de un sistema

$$
\left\{\begin{array}{l}
u_{t}=v, \\
v_{t}=\Delta u-\alpha(x) u-f(u)-a(x) v,
\end{array}\right.
$$

que, a su vez. puede ser enmarcado en un sistema semilineal abstracto

$$
\left\{\begin{array}{l}
\frac{d U}{d t}+A U=F(U), \\
U(0)=U_{0}
\end{array}\right.
$$

donde $U=\left(\begin{array}{c}u \\ v\end{array}\right), F(U)=\left(\begin{array}{c}0 \\ -f(u)-a(x) v\end{array}\right)$ y el operador $A$ definido por

$$
A U=\left(\begin{array}{cc}
0 & -I \\
(-\Delta+\alpha(\cdot)) I & 0
\end{array}\right)\left(\begin{array}{l}
u \\
v
\end{array}\right)=\left(\begin{array}{cc}
0 & -v \\
-\Delta u+\alpha(x) u & 0
\end{array}\right)
$$

con dominio $D(A)=H^{2}\left(\mathbb{R}^{n}\right) \times H^{1}\left(\mathbb{R}^{n}\right)$.

Afirmación 3.1. El operador $A: D(A) \subset H \rightarrow H$ es un operador maximal monótono en $D(A)$.

\section{En Efecto,}

i) $A$ monótono: Sea $U=\left(\begin{array}{c}u \\ v\end{array}\right) \in D(A)$, se tiene

$$
\begin{aligned}
(A U, U)_{H} & =\left(A\left(\begin{array}{c}
u \\
v
\end{array}\right),\left(\begin{array}{c}
u \\
v
\end{array}\right)\right) \\
& =\left(\left(\begin{array}{c}
-v \\
-\Delta u+\alpha(x) u
\end{array}\right),\left(\begin{array}{c}
u \\
v
\end{array}\right)\right) \\
& =\int_{\mathbb{R}^{n}}[-\nabla v \cdot \nabla u-\alpha(x) v u+((-\Delta u)+\alpha(x) u) v] d x \\
& =-\int_{\mathbb{R}^{n}}(\nabla v \cdot \nabla u+(\Delta u) v) d x=0 \geq 0 .
\end{aligned}
$$

ii) $A$ maximal: Sea $F=\left(\begin{array}{c}f \\ g\end{array}\right) \in H$ debemos demostrar que existe una única $D(A)$ tal que $\left(\begin{array}{l}u \\ v\end{array}\right)+A\left(\begin{array}{l}u \\ v\end{array}\right)=\left(\begin{array}{l}f \\ g\end{array}\right)$.

En efecto, de la igualdad

$$
\left(\begin{array}{l}
u \\
v
\end{array}\right)+A\left(\begin{array}{l}
u \\
v
\end{array}\right)=\left(\begin{array}{l}
f \\
g
\end{array}\right)
$$

se tiene

$$
\left\{\begin{aligned}
u-v & =f \\
v-\Delta u+\alpha(x) u & =g
\end{aligned}\right.
$$

Sumando se obtiene

$$
-\Delta u+(\alpha(x)+1) u=f+g \text { donde } f+g \in L^{2}\left(\mathbb{R}^{n}\right) .
$$


Definamos la aplicación $a: H^{1}\left(\mathbb{R}^{n}\right) \times H^{1}\left(\mathbb{R}^{n}\right) \rightarrow \mathbb{R}$ dada por

$$
a(u, v)=(\nabla u, \nabla v)_{L^{2}\left(\mathbb{R}^{n}\right)}+((\alpha(\cdot)+1) \cdot v)_{L^{2}\left(\mathbb{R}^{n}\right)}
$$

y cumple lo siguiente

a) a es una forma bilineal; debido a que el producto interno es bilineal en $L^{2}\left(\mathbb{R}^{n}\right)$.

b) $a$ es continua

En Efecto,

$$
\begin{aligned}
& |a(u, v)|=\mid(\nabla u, \nabla v)+((\alpha(\cdot)+1) u, v)) \mid \\
& =|(\nabla u, \nabla v)+(\alpha(\cdot) u, v)+(u, v)| \\
& =\left|(u, v)_{H^{1}\left(\mathbb{R}^{n}\right)}+(\alpha(\cdot) u, v)\right| \\
& \leq\left|(u, v)_{H^{1}\left(\mathbb{R}^{n}\right)}\right|+|(\alpha(\cdot) u, v)| \\
& \leq|u|_{H^{1}\left(\mathbb{R}^{n}\right)}|v|_{H^{1}\left(\mathbb{R}^{n}\right)}+|\alpha|_{\infty}|u|_{H^{1}\left(\mathbb{R}^{n}\right)}|v|_{H^{1}\left(\mathbb{R}^{n}\right)} \\
& \leq\left(1+|\alpha|_{\infty}\right)|u|_{H^{1}\left(\mathbb{R}^{n}\right)}|v|_{H^{1}\left(\mathbb{R}^{n}\right)} \text {. }
\end{aligned}
$$

c) $a$ es coerciva

En Efecto,

$$
\begin{aligned}
|a(u, u)| & =\mid(\nabla u, \nabla u)+((\alpha(\cdot)+1) u, u)) \mid \\
& =|\nabla u|_{L^{2}\left(\mathbb{R}^{n}\right)}^{2}+|u|_{L^{2}\left(\mathbb{R}^{n}\right)}^{2}+|(\alpha(\cdot) u, u)| \\
& \geq|\nabla u|_{L^{2}\left(\mathbb{R}^{n}\right)}^{2}+|u|_{L^{2}\left(\mathbb{R}^{n}\right)}^{2}+\alpha_{0}|u|_{L^{2}\left(\mathbb{R}^{n}\right)}^{2} \\
& \geq|\nabla u|_{L^{2}\left(\mathbb{R}^{n}\right)}^{2}+\left(1+\alpha_{0}\right)|u|_{L^{2}\left(\mathbb{R}^{n}\right)}^{2} \\
& \geq C|u|_{H^{1}\left(\mathbb{R}^{n}\right)}^{2}
\end{aligned}
$$

donde $C=\min \left\{1, \alpha_{0}+1\right\}$.

Dado $\psi \in L^{2}\left(\mathbb{R}^{n}\right)$, definimos el funcional

$$
\begin{aligned}
L: H^{1}\left(\mathbb{R}^{n}\right) & \rightarrow \mathbb{R} \\
v & \mapsto\langle L, v\rangle:=(\psi, v)
\end{aligned}
$$

entonces $L$ es una forma lineal y continua, esto es, $L \in\left(H^{1}\left(\mathbb{R}^{n}\right)^{\prime}\right.$. Para está $L$. debido al teorema de Lax - Milgram (Brezis [2]) existe una única $u \in H^{1}\left(\mathbb{R}^{n}\right)$ tal que

$$
a(u, v)=\langle L, v\rangle:=(\psi, v)_{L^{2}\left(\mathbb{R}^{n}\right)} ; \quad \forall v \in H^{1}\left(\mathbb{R}^{n}\right) .
$$

Para todo $\varphi \in D\left(\mathbb{R}^{n}\right)$, tenemos

$$
\begin{aligned}
(\psi, \varphi)_{L^{2}\left(\mathbb{R}^{n}\right)} & =\langle L, \varphi\rangle \\
& =a(u, \varphi) \\
& =(\nabla u, \nabla \varphi)+((\alpha(\cdot)+1) u, \varphi) \\
& =(-\Delta u, \varphi)+((\alpha(\cdot)+1) u, \varphi) \\
& =(-\Delta u+(\alpha(\cdot)+1) u, \varphi) .
\end{aligned}
$$

Luego $-\Delta u+(\alpha(\cdot)+1) u=\psi \in L^{2}\left(\mathbb{R}^{n}\right)$ y por el teorema 3.3.1 (Kesavan [5]) resulta que $u \in H^{2}\left(\mathbb{R}^{n}\right)$. Por lo tanto dado $F=\left(\begin{array}{c}f \\ g\end{array}\right) \in H=H^{1}\left(\mathbb{R}^{n}\right) \times L^{2}\left(\mathbb{R}^{n}\right)$ existe una única $\left(\begin{array}{c}u \\ v\end{array}\right) \in D(A)=H^{2}\left(\mathbb{R}^{n}\right) \times H^{1}\left(\mathbb{R}^{n}\right)$ tal que $\left(\begin{array}{c}u \\ v\end{array}\right)+A\left(\begin{array}{c}u \\ v\end{array}\right)=\left(\begin{array}{c}f \\ g\end{array}\right)$, esto prueba que $A$ es maximal.

Afirmación 3.2. El operador $F: H \rightarrow H$ dado por

$$
F(U)=\left(\begin{array}{c}
0 \\
f(u)+a(x) v
\end{array}\right) \text { para todo } U=\left(\begin{array}{l}
u \\
v
\end{array}\right) \in H
$$


está bien definido.

\section{En efecto,}

Sea $U=\left(\begin{array}{l}u \\ v\end{array}\right) \in H$ solo nos faltaría comprobar que $f(u)+a(x) v \in L^{2}\left(\mathbb{R}^{n}\right)$.

Por las hipótesis (H2), (H3) y de la desigualdad $(a+b)^{2} \leq 2\left(a^{2}+b^{2}\right)$ se tiene:

$$
\begin{aligned}
\int_{\mathbb{R}^{n}}|f(u)|^{2} d x & \leq \int_{\mathbb{R}^{n}}\left[\left(1+|u|^{p-1}\right)|u|\right]^{2} d x \\
& \leq 2 C\left(\int_{\mathbb{R}^{n}}|u|^{2} d x+\int_{\mathbb{R}^{n}}|u|^{2 p} d x\right) \\
& =2 C\left(|u|_{L^{2}\left(\mathbb{R}^{n}\right)}^{2}+|u|_{L^{2 p}\left(\mathbb{R}^{n}\right)}^{2 p}\right) .
\end{aligned}
$$

De la inmersión de Sobolev $H^{1}\left(\mathbb{R}^{n}\right) \hookrightarrow L^{2 p}\left(\mathbb{R}^{n}\right),(n-2) p \leq n$ (Kesavan [5]) existe una constante $\tilde{C}$ tal que

$$
|u|_{L^{2 p}\left(\mathbb{R}^{n}\right)} \leq \tilde{C}|u|_{H^{1}\left(\mathbb{R}^{n}\right)} \text {, para todo } u \in H^{1}\left(\mathbb{R}^{n}\right) .
$$

De las desigualdades (10) y (11) se obtiene

$$
\int_{\mathbb{R}^{n}}|f(u)|^{2} d x \leq 2 C\left(|u|_{L^{2}\left(\mathbb{R}^{n}\right)}^{2}+\tilde{C}|u|_{H^{1}\left(\mathbb{R}^{n}\right)}^{2 p}\right)<\infty .
$$

Además de la hipótesis (H1) se tiene

$$
\int_{\mathbb{R}^{n}}|a(x) v|^{2} d x \leq|a|_{\infty}^{2} \int_{\mathbb{R}^{n}}|v|^{2} d x<\infty .
$$

Por lo tanto de (12) y (13) se prueba la buena definición de $F$.

Afirmación 3.3. El operador $F: H \rightarrow H$ es localmente lipschitziano.

\section{En efecto,}

Sean $U_{1}=\left(\begin{array}{l}u_{1} \\ v_{1}\end{array}\right) \in H$ y $U_{2}=\left(\begin{array}{c}u_{2} \\ v_{2}\end{array}\right) \in H$ tal que

$$
\left\|U_{1}\right\|_{H} \leq M \text { y }\left\|U_{2}\right\|_{H} \leq M \text { para algún } M>0 .
$$

Se tiene que

$$
\begin{aligned}
\left\|F\left(U_{1}\right)-F\left(U_{2}\right)\right\|_{H} & =\left\|\left(\begin{array}{c}
0 \\
\leq
\end{array}\right)\right\|_{H} \\
\leq & \left.\mid f\left(u_{1}\right)+a(x) v_{1}-f\left(u_{2}\right)-a(x) v_{2}\right)\left.\right|_{L^{2}\left(\mathbb{R}^{n}\right)}+\left|a(x)\left(v_{1}-v_{2}\right)\right|_{L^{2}\left(\mathbb{R}^{n}\right)}^{2} \\
\leq & C^{2} \int_{\mathbb{R}^{n}}\left\{\left(1+\left|u_{1}\right|^{p-1}+\left|u_{2}\right|^{p-1}\right)\left|u_{1}-u_{2}\right|\right\}^{2} d x+|a|_{\infty}^{2}\left|v_{1}-v_{2}\right|_{L^{2}\left(\mathbb{R}^{n}\right)}^{2} \\
\leq & 3 C^{2}\left(\int_{\mathbb{R}^{n}}\left|u_{1}-u_{2}\right|^{2} d x+\int_{\mathbb{R}^{n}}\left|u_{1}\right|^{2(p-1)}\left|u_{1}-u_{2}\right|^{2} d x\right. \\
& \left.\quad+\int_{\mathbb{R}^{n}}\left|u_{2}\right|^{2(p-1)}\left|u_{1}-u_{2}\right|^{2} d x\right)+|a|_{\infty}^{2}\left|v_{1}-v_{2}\right|_{L^{2}\left(\mathbb{R}^{n}\right)}^{2} .
\end{aligned}
$$

Como $\frac{1}{p}+\frac{1}{\frac{p}{p-1}}=1$, usandó la desigualdad de Hölder obtenemos

$$
\begin{aligned}
\left\|F\left(U_{1}\right)-F\left(U_{2}\right)\right\|_{H} \leq & 3 C^{2}\left(\left|u_{1}-u_{2}\right|_{L^{2}\left(\mathbb{R}^{n}\right)}^{2}+\left|u_{1}\right|_{L^{2 p}\left(\mathbb{R}^{n}\right)}^{2(p-1)}\left|u_{1}-u_{2}\right|_{L^{2 p}\left(\mathbb{R}^{n}\right)}^{2}\right. \\
& \left.\left|u_{2}\right|_{L^{2 p}\left(\mathbb{R}^{n}\right)}^{2(p-1)}\left|u_{1}-u_{2}\right|_{L^{2 p}\left(\mathbb{R}^{n}\right)}^{2}\right)+|a|_{\infty}^{2}\left|v_{1}-v_{2}\right|_{L^{2}\left(\mathbb{R}^{n}\right)}^{2} .
\end{aligned}
$$


De las desigualdades (11) y (14) obtenemos

$$
\begin{aligned}
\left\|F\left(U_{1}\right)-F\left(U_{2}\right)\right\|_{H} \leq & 3 C^{2}\left(\left|u_{1}-u_{2}\right|_{L^{2}\left(\mathbb{R}^{n}\right)}^{2}+\tilde{C}^{2 p} M^{2(p-1)}\left|u_{1}-u_{2}\right|_{H^{1}\left(\mathbb{R}^{n}\right)}^{2}\right. \\
& \left.\quad+\tilde{C}^{2 p} M^{2(p-1)}\left|u_{1}-u_{2}\right|_{H^{1}\left(\mathbb{R}^{n}\right)}^{2}\right)+|a|_{\infty}^{2}\left|v_{1}-v_{2}\right|_{L^{2}\left(\mathbb{R}^{n}\right)}^{2} \\
\leq & L_{M}\left(\left|u_{1}-u_{2}\right|_{H^{1}\left(\mathbb{R}^{n}\right)}^{2}+\left|v_{1}-v_{2}\right|_{L^{2}\left(\mathbb{R}^{n}\right)}^{2}\right) \\
\leq & L_{M}|| U_{1}-U_{2} \|_{H}
\end{aligned}
$$

donde $L_{M}=\max \left\{3 C^{2}\left(1+\tilde{C}^{2 p} M^{2(p-1)}\right),|a|_{\infty}^{2}\right\}$.

Como $F$ es localmente lipschitziana y $U_{0}=\left(\begin{array}{c}u_{0} \\ v_{0}\end{array}\right) \in D(A)$, por la proposición 4.3.9 (Cazenave [4]) se tiene que existe

$$
U=\left(\begin{array}{c}
u \\
u_{t}
\end{array}\right) \in C\left(\left[0, T_{\max }\left[; H^{2}\left(\mathbb{R}^{n}\right) \times H^{1}\left(\mathbb{R}^{n}\right)\right) \cap C^{1}\left(\left[0, T_{\max }\left[; H^{1}\left(\mathbb{R}^{n}\right) \times L^{2}\left(\mathbb{R}^{n}\right)\right)\right.\right.\right.\right.
$$

solución clásica de (1), de donde resulta

$$
u \in C\left(\left[0, T_{\max }\left[; H^{2}\left(\mathbb{R}^{n}\right)\right) \cap C^{1}\left(\left[0, T_{\max }\left[; H^{1}\left(\mathbb{R}^{n}\right)\right) \cap C^{2}\left(\left[0, T_{\max }\left[; L^{2}\left(\mathbb{R}^{n}\right)\right) .\right.\right.\right.\right.\right.\right.
$$

\section{B) Unicidad de la solución regular}

Consideremos $u$ y $v$ soluciones de (1) y $w=u-v$ se tiene

$$
\left\{\begin{array}{l}
w_{t t}-\Delta w+\alpha(x) w+f(u)-f(v)+a(x) w_{t}=0, \\
w(0)=w_{t}(0)=0 .
\end{array}\right.
$$

Multiplicando la ecuación (15) por $w_{t}$ e integrando sobre $\mathbb{R}^{n}$ obtenemos:

$$
\begin{aligned}
\frac{1}{2} \frac{d}{d t}\left(\left|w_{t}\right|_{L^{2}\left(\mathbb{R}^{n}\right)}^{2}+|\nabla w|_{L^{2}\left(\mathbb{R}^{n}\right)}^{2}+\right. & \left.\left|\alpha^{1 / 2}(\cdot) w\right|_{L^{2}\left(\mathbb{R}^{n}\right)}^{2}\right) \\
& \leq \int_{\mathbb{R}^{n}}|f(u)-f(v)|\left|w_{t}\right| d x+\int_{\mathbb{R}^{n}}|a(x)|\left|w_{t}\right|^{2} d x .
\end{aligned}
$$

Por la hipótesis (H1) se obtiene

$$
\begin{aligned}
\frac{1}{2} \frac{d}{d t}\left(\left|w_{t}\right|_{L^{2}\left(\mathbb{R}^{n}\right)}^{2}+|\nabla w|_{L^{2}\left(\mathbb{R}^{n}\right)}^{2}+\left|\alpha^{1 / 2}(\cdot) w\right|_{L^{2}\left(\mathbb{R}^{n}\right)}^{2}\right) & \\
& \leq \int_{\mathbb{R}^{n}}\left|f(u)-f(v) \| w_{t}\right| d x+|a|_{\infty}\left|w_{t}\right|_{L^{2}\left(\mathbb{R}^{n}\right)}^{2} .
\end{aligned}
$$

De la primera integral del segundo miembro y de la hipótesis (H3), se obtiene

$$
\begin{aligned}
\int_{\mathbb{R}^{n}}|f(u)-f(v)|\left|w_{t}\right| d x & \leq C \int_{\mathbb{R}^{n}}\left(1+|u|^{p-1}+|v|^{p-1}\right)|u-v \|| w_{t} \mid d x \\
& =C \int_{\mathbb{R}^{n}}\left(1+|u|^{p-1}+|v|^{p-1}\right)|w|\left|w_{t}\right| d x \\
& =C\left(\int_{\mathbb{R}^{n}}|w|\left|w_{t}\right| d x+\int_{\mathbb{R}^{n}}|u|^{p-1}|w|\left|w_{t}\right| d x\right. \\
& \left.+\int_{\mathbb{R}^{n}}|v|^{p-1}|w|\left|w_{t}\right| d x\right) .
\end{aligned}
$$

Como $\frac{p-1}{2 p}+\frac{1}{2 p}+\frac{1}{2}=1$, por la desigualdad de Hölder generalizado se obtiene

$$
\int_{\mathbb{R}^{n}}|f(u)-f(v)|\left|w_{t}\right| d x \leq C\left(|w|_{L^{2}\left(\mathbb{R}^{n}\right)}\left|w_{t}\right|_{L^{2}\left(\mathbb{R}^{n}\right)}+m(t)|w|_{L^{2 p}\left(\mathbb{R}^{n}\right)}\left|w_{t}\right|_{L^{2}\left(\mathbb{R}^{n}\right)}\right)
$$


donde $m(t)=|u|_{L^{2 p}\left(\mathbb{R}^{n}\right)}^{p-1}+|v|_{L^{2 p}\left(\mathbb{R}^{n}\right)}^{p-1}$.

De la desigualdad (11) se tiene

$$
\int_{\mathbb{R}^{n}}|f(u)-f(v)|\left|w_{t}\right| d x \leq C\left(|w|_{H^{1}\left(\mathbb{R}^{n}\right)}\left|w_{t}\right|_{L^{2}\left(\mathbb{R}^{n}\right)}+\tilde{C}^{2} m(t)|w|_{H^{1}\left(\mathbb{R}^{n}\right)}\left|w_{t}\right|_{L^{2}\left(\mathbb{R}^{n}\right)}\right) .
$$

Usando la desigualdad $2 a b \leq a^{2}+b^{2}$ se obtiene

$$
\int_{\mathbb{R}^{n}}|f(u)-f(v)|\left|w_{t}\right| d x \leq C \tilde{C}^{2} m(t)\left(|w|_{H^{1}\left(\mathbb{R}^{n}\right)}^{2}+\left|w_{t}\right|_{L^{2}\left(\mathbb{R}^{n}\right)}^{2}\right) .
$$

De las desigualdades (16) y (17) se tiene

$$
\frac{1}{2} \frac{d}{d t}\left(\left|w_{t}\right|_{L^{2}\left(\mathbb{R}^{n}\right)}^{2}+|\nabla w|_{L^{2}\left(\mathbb{R}^{n}\right)}^{2}+\left|\alpha^{1 / 2}(\cdot) w\right|_{L^{2}\left(\mathbb{R}^{n}\right)}^{2}\right) \leq \widehat{C} m(t)\left(|w|_{H^{1}\left(\mathbb{R}^{n}\right)}^{2}+\left|w_{t}\right|_{L^{2}\left(\mathbb{R}^{n}\right)}^{2}\right) .
$$

Integrando desde 0 hasta $t$ se tiene

$$
\left|w_{t}\right|_{L^{2}\left(\mathbb{R}^{n}\right)}^{2}+|\nabla w|_{L^{2}\left(\mathbb{R}^{n}\right)}^{2}+\left|\alpha^{1 / 2}(\cdot) w\right|_{L^{2}\left(\mathbb{R}^{n}\right)}^{2} \leq \widehat{C} \int_{0}^{t} m(t)\left(|w|_{H^{1}\left(\mathbb{R}^{n}\right)}^{2}+\left|w_{t}\right|_{L^{2}\left(\mathbb{R}^{n}\right)}^{2}\right) d x .
$$

Como $\alpha(x) \geq \alpha_{0}$, mayorando por la izquierda en la desigualdad precedente

$$
|w|_{H^{1}\left(\mathbb{R}^{n}\right)}^{2}+\left|w_{t}\right|_{L^{2}\left(\mathbb{R}^{n}\right)}^{2} \leq \frac{\widehat{C}}{K} \int_{0}^{t} m(t)\left(|w|_{H^{1}\left(\mathbb{R}^{n}\right)}^{2}+\left|w_{t}\right|_{L^{2}\left(\mathbb{R}^{n}\right)}^{2}\right) d x
$$

donde $K=\min \left\{1, \alpha_{0}\right\}$.

Por la desigualdad de Gronwall, resulta

$$
|w|_{H^{1}\left(\mathbb{R}^{n}\right)}^{2}+\left|w_{t}\right|_{L^{2}\left(\mathbb{R}^{n}\right)}^{2}=0 .
$$

Luego $w=0$, es decir, $u=v$.

\section{C) Prolongamiento de la solución regular}

Si multiplicamos la ecuación (1) por $u_{t}$ e integrando sobre $\mathbb{R}^{n}$, obtenemos:

$$
\frac{1}{2} \frac{d}{d t}\left(\int_{\mathbb{R}^{n}}\left[|\nabla u|^{2}+\left|u_{t}\right|^{2}+\alpha(x) u^{2}\right] d x\right)+\int_{\mathbb{R}^{n}} f(u) u_{t} d x+\int_{\mathbb{R}^{n}} a(x) u_{t}^{2} d x=0 .
$$

Por la hipótesis (H2) la función $F(s)=\int_{0}^{s} f(t) d t, \forall s \in \mathbb{R}$, está bien definida. Luego

$$
\frac{1}{2} \frac{d}{d t}\left(\int_{\mathbb{R}^{n}}\left[|\nabla u|^{2}+\left|u_{t}\right|^{2}+\alpha(x) u^{2}\right] d x+\int_{\mathbb{R}^{n}} F(u) d x\right)=-\int_{\mathbb{R}^{n}} a(x) u_{t}^{2} d x .
$$

Definimos la energía asociada al sistema (1) como

$$
E(t):=\frac{1}{2} \int_{\mathbb{R}^{n}}\left[|\nabla u(x, t)|^{2}+\left|u_{t}(x, t)\right|^{2}+\alpha(x)|u(x, t)|^{2}\right] d x+\int_{\mathbb{R}^{n}} F(u(x, t)) d x .
$$

Luego de la ecuación (18) se tiene

$$
\frac{d}{d t} E(t)=-\int_{\mathbb{R}^{n}} a(x)\left|u_{t}(x, t)\right|^{2} d x \leq 0,
$$

lo que demuestra que la energía asociada al sistema (1) es no creciente, para todo $t \in[0,+\infty)$.

Afirmación 3.4. $T_{\max }=+\infty$. 
En efecto, si suponemos que $T_{\max }<+\infty$ entonces por el teorema 4.3.4 (Cazenave [4]) se tiene

$$
\lim _{t \rightarrow t_{\text {max }}}\|U(t)\|_{H}=+\infty .
$$

Pero

$$
\begin{aligned}
\|U(t)\|_{H}=\int_{\mathbb{R}^{n}}\left[|\nabla u|^{2}+\left|u_{t}\right|^{2}+\alpha(x)|u|^{2}\right] d x & \leq E(t) \\
& \leq E(0)<\infty,
\end{aligned}
$$

lo cual es absurdo. Por lo tanto $T_{\max }=+\infty$. Luego, la solución regular existen en todo el intervalo $[0,+\infty)$.

\section{Conclusión}

Con la teoría de semigrupos se logra demostrar que el problema (1), con las hipótesis (H1) - (H4) y datos iniciales $\left(u_{0}, u_{1}\right) \in H^{2}\left(\mathbb{R}^{n}\right) \times H^{1}\left(\mathbb{R}^{n}\right)$ tiene una única solución fuerte $u:[0,+\infty) \rightarrow \mathbb{R}$ tal que

$$
u \in C\left([0,+\infty) ; H^{2}\left(\mathbb{R}^{n}\right)\right) \cap C^{1}\left([0,+\infty) ; H^{1}\left(\mathbb{R}^{n}\right)\right) \cap C^{2}\left([0,+\infty) ; L^{2}\left(\mathbb{R}^{n}\right)\right) .
$$

Además la energía asociada al problema (1) es una función no creciente en el intervalo $[0,+\infty)$.

Más aún con los mismos argumentos realizados en el presente trabajo se puede demostrar, con las hipótesis (H1) - (H4) y datos iniciales $\left(u_{0}, u_{1}\right) \in H^{1}\left(\mathbb{R}^{n}\right) \times L^{2}\left(\mathbb{R}^{n}\right)$, que el problema (1) tiene una solución débil en el espacio

$$
u \in C\left([0,+\infty) ; H^{1}\left(\mathbb{R}^{n}\right)\right) \cap C^{1}\left([0,+\infty) ; L^{2}\left(\mathbb{R}^{n}\right)\right)
$$




\section{REFERENCIAS BIBLIOGRÁFICAS}

[1] Adams, R. A. Sobolev Spaces. New York; Academic Press, 1975.

[2] Brézis, H. Analyse Fonctionnelle - Théorie et Applications. Paris: Mason, 1973.

[3] Cabanillas Lapa, E. Estabilización de la energía para una ecuación de Kirchoff con disipación localizada. Revista PESQUIMAT, Vol VII N 1. FCM-UNMSM, 2004.

4] Haraux A., Cazenave T. An Introduction to Semilinear Evolution Equations, Oxford Lecture Series in Mathematics and its Applications, 13, Oxford University Press, 1998.

5] Kesavan. S. Topics in Functional Analysis and Applications. New Delhi: Willey Easten Limited, 1990.

[6] Muñoz R., J. Bisognin V. Exponential decay to partially thermoelaste materials. LNCC RJ Brasil, 1996.

[7] Pazy A. Semigrupo of linear Operators and applications to partial differential equations. Springer Verlag. New York, 1993.

[8] Peña Miranda Carlos A. Comportamiento asintótico para la ecuación de onda semilineal con amortiguamiento local en dominios no acotado, Tesis Maestria UNMSM Perú, 2012.

[9] Pérez Salvatierra, A. Decaimento de soluções de equações parcialmente Viscoláelasticos, Tesis doctorado UFRJ Brasil, 1997.

[10] Pérez Salvatierra, A. Decaimiento Exponencial para materiales parcialmente termoelástico, Revista PESQUIMAT, Vol IV N², pp 1 - 6. FCM-UNMSM, 2001.

111 Pérez Salvatierra, A. Decaimientos exponencial de la ecuación de onda con potencial y amortiguamiento localmente distribuido, Revista PESQUIMAT, Vol VII $\mathrm{N}^{\circ}$ 1. FCM-UNMSM, 2004.

[12] Portillo Oquendo, H. Muñoz R. J. Sobre um problema de contacto unidimensional de ondas elásticas localmente amortecidas, SBA 46. Seminário Brasileiro de Analise, 1997.

113| Zuazua, E. Exponential decay for the semilinear wave equations, with locally distributed damping, Commu. Partial Diff. Equations, 15 (2), pp 205 - 235, 1990.

[14] Zuazua, E. Exponential decay for the semilinear wave equations, with localized Damping in Unbounded Domains, J. Math Pures et Appl., 70, pp 513 - 529, 1991. 